\title{
ANTIANDROGENIC THERAPY WITH CIPROTERONE ACETATE IN FEMALE PATIENTS WHO SUFFER FROM BOTH ANDROGENETIC ALOPECIA AND ACNE VULGARIS
}

\author{
ANDREI CONEAC ${ }^{1}$, ADRIANA MURESAN ${ }^{2}$, MEDA SANDRA ORASAN ${ }^{3}$ \\ ${ }^{1}$ Department of Histology, Morphological Sciences Division, Iuliu Haţieganu \\ University of Medicine and Pharmacy, Cluj-Napoca, Romania \\ ${ }^{2}$ Department of Physiology, Physiological Sciences Division, Iuliu Haţieganu \\ University of Medicine and Pharmacy, Cluj-Napoca, Romania \\ ${ }^{3}$ Department of Pathophysiology, Physiological Sciences Division, Iuliu Haţieganu \\ University of Medicine and Pharmacy, Cluj-Napoca, Romania
}

\begin{abstract}
Background. Androgenetic Alopecia in Women (AGA) occurs due to an underlying susceptibility of hair follicles to androgenic miniaturization, caused by androgens. Clinically, AGA is characterized by progressive hair loss, with a marked hair thinning in the fronto-parietal area so that the scalp can be easily seen.

Acne vulgaris is androgen-dependent and often affects the skin that has an increased number of oil glands: face, back and chest. Although the sebaceous glands are present on the scalp too, it is very rare to get acne at this site, as the hair acts as a wig and allows the sebum to drain and does not block the pores.

Both AGA and Acne Vulgaris are signs of hyperandrogenism. Cyproterone acetate/ethinyl estradiol $(2 \mathrm{mg} / 0.035 \mathrm{mg})$ products are authorized for the treatment of androgenetic symptoms in women, such as acne, seborrhea, mild forms of hirsutism and androgenetic alopecia.

Our study had a double purpose:

- To evaluate the result of the study regimen Melleva 35 (one pill per day, for 3 consecutive months) in patients with moderate to severe acne, suffering also from Androgenetic Alopecia;

- To establish the efficacy of the drug on acne and alopecia improvement, both from the doctor's and patient's point of view.

Patients and methods. After being informed of the aims and procedures of the study, participants provided a written informed consent. A number of 35 female subjects with moderate to severe acne vulgaris remained in the study. The subjects had also been diagnosed as suffering from AGA, on the basis of clinical criteria, including the pattern of hair loss and trichoscopy assessment.

Results. $83 \%$ of study subjects reported that their hair did not continue to fall after 3 months of antiandrogen therapy. The females were evaluated using trichoscopy and the doctor noticed hair regrowth in $77 \%$ of the cases. Regarding the improvement of acne lesions after the treatment, 40\% of study subjects recorded good improvement and $26 \%$ recorded excellent results with Melleva 35.

The acceptance of the treatment was very high, $86 \%$ patients were compliant with the study therapy. The rate of adverse events (5 cases) was within the limits of the treatment tested by the study.

Almost a third of the total number of subjects (28.5\%) reached a good satisfaction level after the treatment, while $37.1 \%$ claimed moderate satisfaction.

Conclusion. There was no correlation between the age of the subjects and the treatment for acne therefore our first hypothesis was rejected.

As a conclusion, antiandrogenic therapy with Melleva 35, 1 pill per day, for 3 consecutive months, shows good results for patients who suffer from both Androgenetic Alopecia and Acne Vulgaris.
\end{abstract}

Keywords: Antiandrogenic therapy, Ciproterone acetate, Androgenetic Alopecia, Acne Vulgaris, Female Pattern Hairloss 


\section{Introduction}

Androgenetic Alopecia in Women (AGA), also known as FPHL (female pattern hair loss), is a non-scarring alopecia which occurs due to an underlying susceptibility of hair follicles to androgenic miniaturization, caused by androgens [1]. It affects about one third of women, being usually noticed after menopause, although it may emerge as early as puberty. Clinically AGA is characterized by progressive hair loss, with a marked hair thinning in the fronto-parietal area so that the scalp can be easily seen [2].

Androgenetic (pattern) baldness is the most common form of alopecia, affecting $50 \%$ of male patients [1]. Braun Falco estimates that $10 \%$ of the women aged up to 30 , and $50 \%$ aged up to 50 suffer from androgenetic alopecia (AGA) [3]. The ratio grows up to $75 \%$ in women above 65 years old [4].

Acne vulgaris (or simply acne) is a multifactorial disease which usually occurs during puberty. It is androgendependent and often affects skin with increased number of oil glands. It is characterized by areas of seborrhea (scaly red skin), comedones (blackheads and whiteheads), papules (pinheads), nodules (large papules), pimples, and possibly scarring [5].

Acne vulgaris is one of the most common dermatological conditions. It is affecting an estimated $80 \%$ of teenagers. At present, two thirds of the women between 30 and 45 years old, especially those living in urban communities suffer from acne [6].

Acne is a disease of the grease glands (sebaceous glands) in the skin and the duct through which its secretions (the sebum) passes to the skin surface. The sites where acne lesions occur are predominantly on the face $(90 \%)$, back $(60 \%)$ and chest $(15 \%)$ [7].

Although the grease glands are present on the scalp too, it is very rare to get acne at this site, as the hair acts as a wig and allows the sebum to drain and does not block the pores [8].

Both FPHL and Acne Vulgaris are signs of hyperandrogenism.

\section{Androgens in acne}

Androgens clearly influence sebaceous glands and sebum production. Androgen receptors are located in both the keratinocytes of the outer root sheath of the hair follicles and the basal layer of the sebaceous gland [9].

The most potent androgens are testosterone and dihydroepiandrosterone, and the combined presence of these hormones increases the size and secretion of sebaceous glands [10]. The average rates of sebum secretion are only slighter higher in males than in females $[11,12]$. The majority of females with acne have serum androgen levels that, although higher, are within normal limits. It is hypothesized that locally produced androgens within the sebaceous gland may contribute to acne $[13,14]$. DHEAS (dehydroepiandrosterone sulfate) may regulate sebaceous gland activity through its conversion to testosterone and DHT within the sebaceous gland. The enzymes required to convert DHEAS to more potent androgens are present within sebaceous glands [15].

\section{Androgens in FPHL}

Androgens and estrogens are the main hormonal regulators involved in FPHL. The hair follicle is sensitive to alterations in circulating estrogen and androgen levels. Androgens have a clear role in determining androgenetic alopecia in males via binding dihydrotestosterone (DHT) to the hair follicle androgen receptors (AR) [16]. In female patients, the role of androgens is not clearly established, and besides heredity, other factors can be involved in the pathophysiology of the disease [17].

In scalp follicles, testosterone is converted to DHT by the enzyme 5 alpha reductase type II. It has been postulated that there is an increased peripheral sensitivity to androgens in FPHL [18].

Many women with FPHL have low levels of circulating sex hormone binding globulin (SHBG), which may increase the available free testosterone at the level of the hair follicle [19].

Although most women with androgenetic alopecia have normal levels of testosterone, this type of alopecia can be a marker of hyperandrogenism in women. Evaluation of patients with FPHL should include clinical assessment for hirsutism, menstrual abnormalities and acne [20]. FPHL can be associated with hyperandrogenic states, while the circulating testosterone levels could be the same in FPHL patients and controls [20].

Cyproterone acetate/ethinyl estradiol $(2 \mathrm{mg} / 0.035$ $\mathrm{mg}$ ) containing medicinal products are authorized for the treatment of androgenetic symptoms in women, such as acne, seborrhea, mild forms of hirsutism and androgenetic alopecia.

\section{Objectives}

- To evaluate the result of the study regimen Melleva in patients with moderate to severe acne, suffering also from Androgenetic Alopecia;

- To establish the efficacy of the drug in relationto the acne and alopecia improvement, both from the doctor and the patient's point of view;

- To identify the adverse reaction rate to the studied drug;

- To evaluate the compliance of female patients to the treatment regimen.

\section{Hypothesis}

We assumed that young patients (15-20 years old) would have better results than those with mature onset of acne and alopecia. 


\section{Material and method \\ Study subjects}

The majority of patients ( 30 women) were recruited from a Private Practice of Dermato-Venerology, located in Cluj-Napoca. Five additional subjects were included by the dermatologists working in the Clinic of Dermatology of the Emergency County Hospital Cluj.

All the subjects selected for the study had been diagnosed as suffering from AGA, on the basis of clinical criteria, including pattern of hair loss and trichoscopy assessment. The study subjects also suffered from moderate to severe acne vulgaris.

In total, 60 patients were selected, out of which seven declined the consent and eighteen females were excluded as they did not fulfill all the inclusion criteria. A number of 35 subjects remained in the study.

\section{Study design}

The survey period was December 2012 to July 2013. The study was conceived to be prospective.

After being informed of the aims and procedures of the study, participants provided a written informed consent. The protocol had been previously approved by the Ethics Committee of the University of Medicine and Pharmacy "Iuliu Hatieganu" Cluj-Napoca.

\section{Inclusion criteria:}

1. Subjects over 18 years old, under the age of 40 ;

2. Females who were not pregnant;

3. Patients suffering from Androgenetic alopecia (AGA) grade I to II on Ludwig's scale;

4. Patients having moderate and severe acne (acne severity equal or over $2(\mathrm{GEA}>2)$ at baseline);

5. Patients for whom the dermatologist concluded that this was the best treatment protocol, after the failure of antibiotic treatment;

6. Females before the onset of menopause;

7. Patients did not take steroids or hormonal medication - contraception pills, for a six months period, before the study.

\section{Exclusion criteria:}

1. Male patients;

2. Patients outside the age range;

3. Females suffering from AGA frontal pattern or III /severe on Ludwig Scale;

4. Patients with mild acne;

5. Pregnancy or breastfeeding;

6. Patients suffering from acute or chronic renal failure or liver diseases;

7. Patients using another hormonal contraceptive;

8. Patients suffering from thrombosis, pulmonary embolism;

9. Patients who had a heart attack or a stroke;

10. Patients with an increased risk of a blood clot in the arteries (diabetes, high blood pressure, high cholesterol or triglycerides);

11. Patients with blood clotting deficiency (protein
C deficiency).

Evaluation methods:

a) for androgenetic alopecia - clinical assessment and trichoscopy;

b) for acne vulgaris;

- Global evaluation of acne (GEA scale);

- Global evaluation of scars (ECCA - simplified version).

\section{Treatment details:}

Melleva 35 ( 2 mg CPA/0.035 mg EE) 1 pill per day, from the first day of the cycle until day 21/each month, for 3 consecutive months.

\section{Results}

Demographic data analysis showed the community distribution for the 35 patients: $71.4 \%$ urban and $28.6 \%$ rural. The mean age of the patients was 25.82 years old.

We structured the results according to the doctor's and patient's evaluations.

\section{Doctor's Evaluation}

Effectiveness of the treatment of Androgenetic Alopecia and the Acne Vulgaris in the study patients (comparison before and after treatment).

\section{A. Improvement of alopecia}

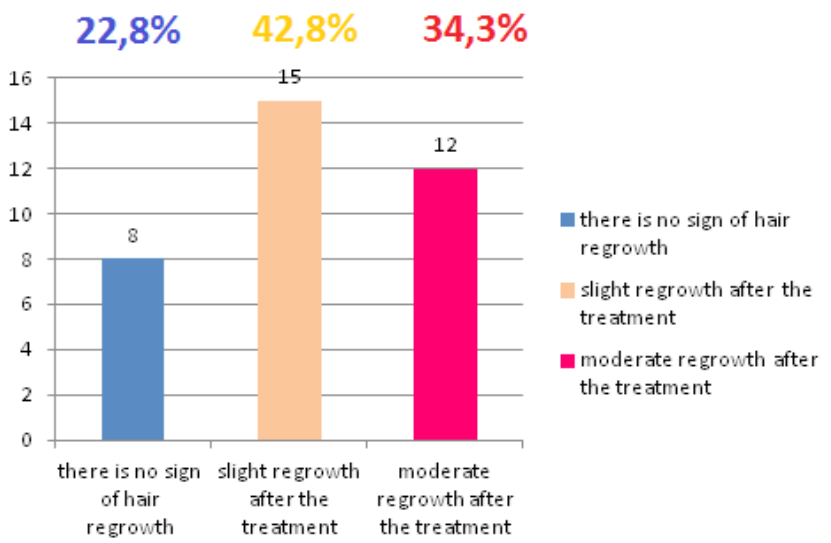

Figure 1. Clinical and trichoscopic assessment of hair regrowth after 3 month of treatment with Melleva 35.

Mean $\pm S T D E V=1.114 \pm 0.758$

Hair regrowth was evaluated both from the clinical and trichoscopic point of view. The trichoscopy (evaluation of the scalp and hair roots by using a handheld device called dermatoscope) is a non-invasive technique which showed that after the 3 months of treatment, patients started having new hair grown over the scalp. Slight regrowth was noticed in $42.8 \%$ of the cases, moderate regrowth in $34.3 \%$ of the females; 8 out of 35 subjects had no signs of hair regrowth at trichoscopy $(22.8 \%)$.

\section{B. Improvement of acne lesions}

The doctor completed the evaluation scales at baseline and end of study (day 90) and compared the:

- Global evaluation of acne (GEA scale); 
- Global evaluation of scars (ECCA - simplified version).

For evaluation purposes we used an original grading, as below:

$0=$ no improvement

$1=$ mild improvement

$2=$ moderate improvement

$3=$ good improvement

$4=$ excellent improvement

Improvements were noticed over the acne lesions and scars after 12 weeks of treatment.

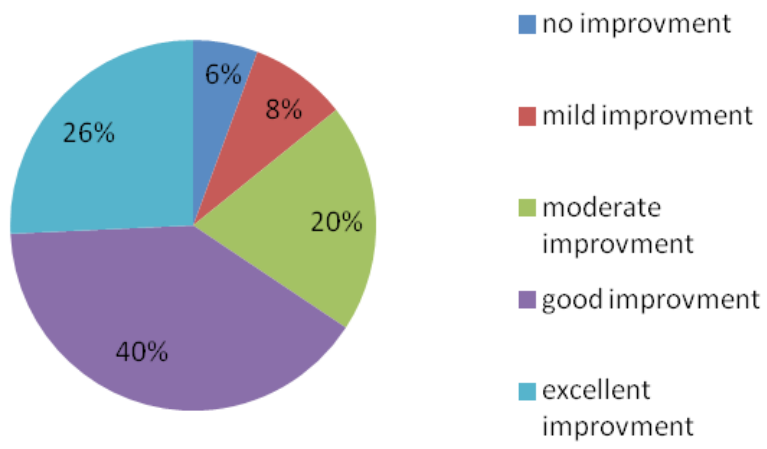

Figure 2. Doctor's evaluation - Improvement of acne lesions after the treatment.

As we can see there was a significant improvement in the majority of female patients, the number of the acne lesions (pustules and papules) and the inflammatory acne was reduced. Only two female patients had no improvement after the treatment $(6 \%)$. Three subjects had mild improvement ( $8 \%$ ), seven patients had moderate improvement (20\%); $40 \%$ of the patients had good improvement after treatment and almost one quarter of the subjects (26\%) recorded excellent results with Melleva 35.

\section{Improvement of acne scars}

More than a half of the total study subjects did not record any improvement $(60 \%)$ representing 21 out of 35 subjects. Six patients recorded mild improvement $(17 \%)$. Four out of the total number, representing $11 \%$, had moderate improvement after the 12 th week of treatment.
There was only one excellent improvement in scars after the treatment (3\%), but good improvements were seen in $9 \%$ of the cases.

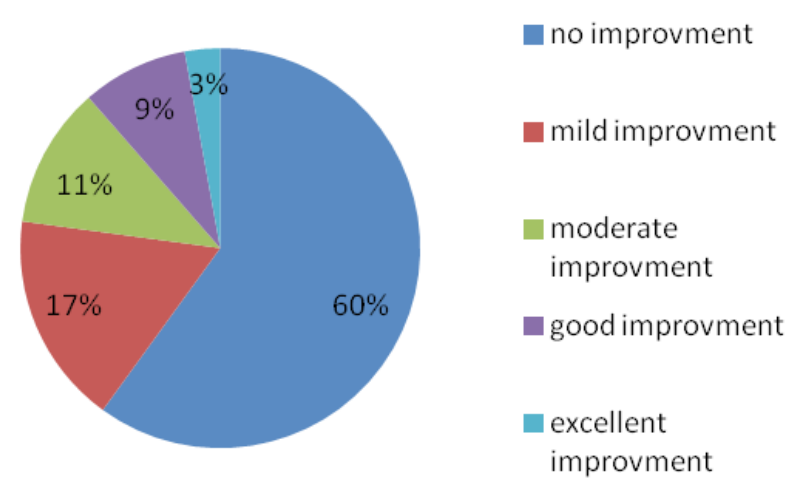

Figure 3. Doctor's evaluation - Improvement of acne scars after the treatment.

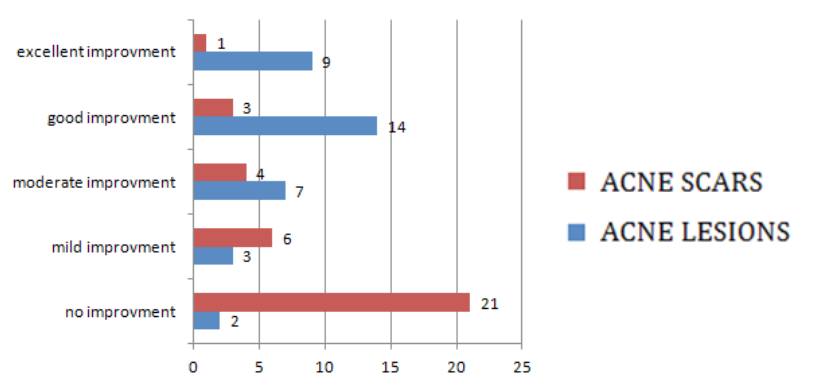

Figure 4. Combined overview of the treatment results on acne lesions and scars

The comparative graph underlines that the acne lesions were better treated than the scars as Melleva therapy worked better on the inflammatory acne lesions than on the scars.

The 35 subjects of the study were divided into two groups on the basis of age and type of acne:

- group A (young patients with adolescent onset of acne): 20 patients;

- group B (mature patients with adult onset of acne):

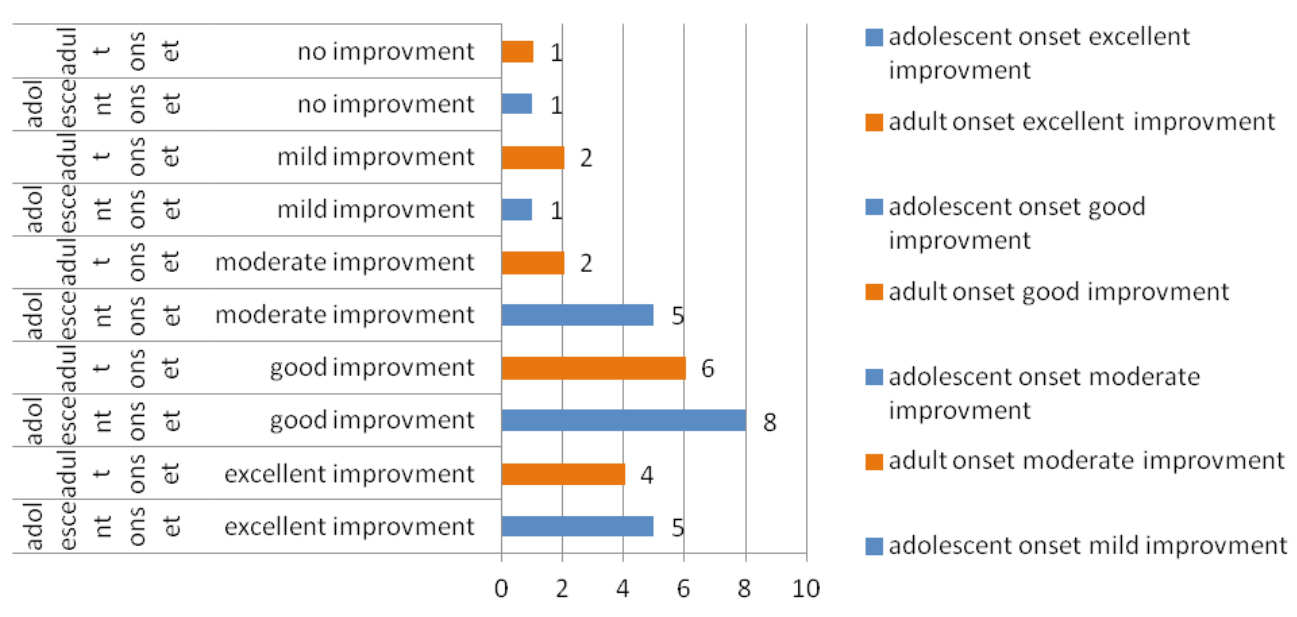

Figure 5. Improvement of acne lesions on the basis of age. 
15 patients.

The graphic representation suggests that the excellent improvement was equal in both age groups, while a good improvement seems to be recorded in the adult group of patients.

In order to see if there was any difference after the treatment for adults or for adolescents, we ran the ChiSquare Test, to see if there was any correlation between the two.

Table 1. Chi-Square test.

\begin{tabular}{lccc}
\hline & Value & Df & $\begin{array}{c}\text { Asymp. Sig. } \\
(2-\text {-sided })\end{array}$ \\
\hline Pearson Chi-Square & $2.946^{\mathrm{a}}$ & 4 & 0.567 \\
Likelihood Ratio & 3.725 & 4 & 0.444 \\
$\begin{array}{l}\text { Linear-by-Linear } \\
\text { Association }\end{array}$ & 0.901 & 1 & 0.342 \\
N of Valid Cases & 35 & & \\
\hline
\end{tabular}

The value of the significance index suggests that our hypothesis is negative, because the value is more than 0.05 . There is no correlation between the age of the subjects and the treatment for acne and our hypothesis was rejected.

\section{Patient's evaluation}

The patient's evaluation consisted of establishing the degree of tolerance, adverse reaction rates, compliance with the treatment for the hair and acne pathology.

\section{a) Hair Loss}

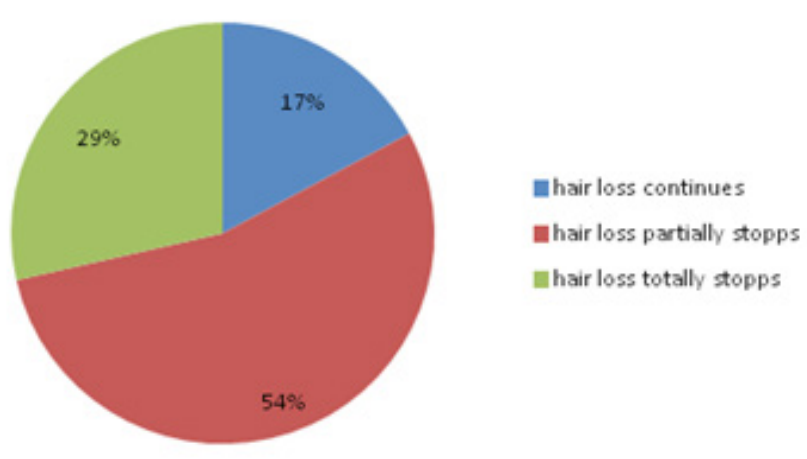

Figure 6. Evaluation of hairloss done by the patient after the treatment.

More than half of the study subjects (54\%) declared that hair loss partially stopped right after the first month of using Melleva therapy. Almost a third of the patients (29\%) reported that hair loss totally stopped under the treatment; $17 \%$ of the females confessed that from their point of view, hair was still falling after the treatment.

\section{b) Acne vulgaris}

Regarding the patients' satisfaction: $5.7 \%$ of total patients declared either excellent satisfaction or no satisfaction. $22.8 \%$ reported mild satisfaction after the treatment, $37.1 \%$ claimed moderate satisfaction.

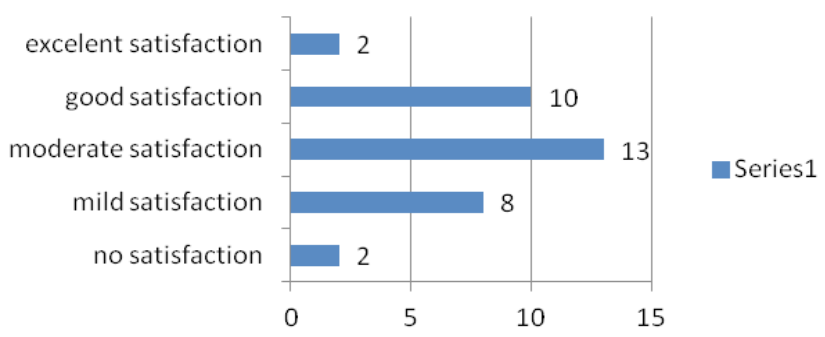

Figure 7. Satisfaction assessment in study subjects after the treatment.

Almost a third of the total number of subjects (28.5\%) reached a good satisfaction level after the treatment.

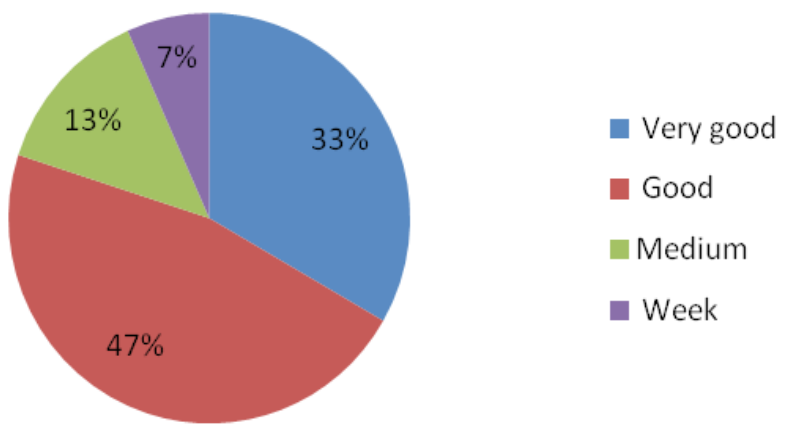

Figure8. Tolerance of treatment in study subjects.

Tolerance was good and very good in $80 \%$ of the cases, with almost an equal distribution between the specified categories; $33 \%$ of the total study subjects recorded a very good tolerance and $47 \%$ (14 females) a good tolerance.

Medium tolerance was encountered in $13 \%$ of the cases, and weak tolerance in $7 \%$ ( 2 patients).

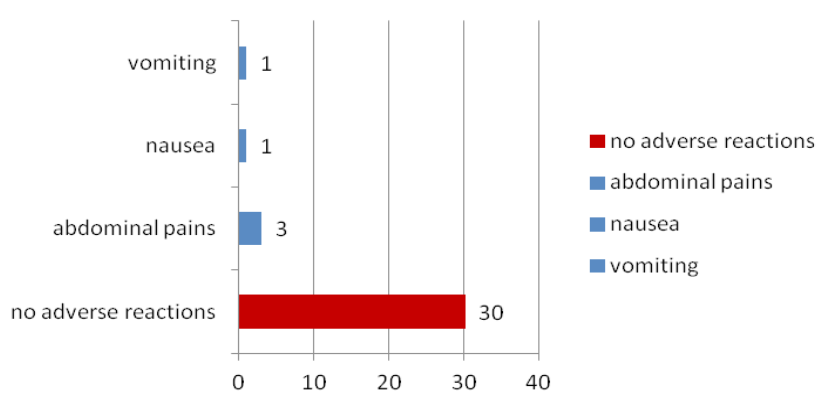

Figure 9. Adverse reactions after treatment.

Five patients out of 35 developed an adverse reaction, representing $14.2 \%$ of the total study subjects. Three female reported abdominal pains in the third month of treatment, one reported nausea and one subject accused vomiting (an episode) in the last month of the trial. The adverse events were treated by symptomatic medication.

As seen in the graph, $86 \%$ patients were compliant to the treatment. The compliance of the patients was very high. Only 5 females (those who recorded adverse reactions) had bad compliance with the treatment. 


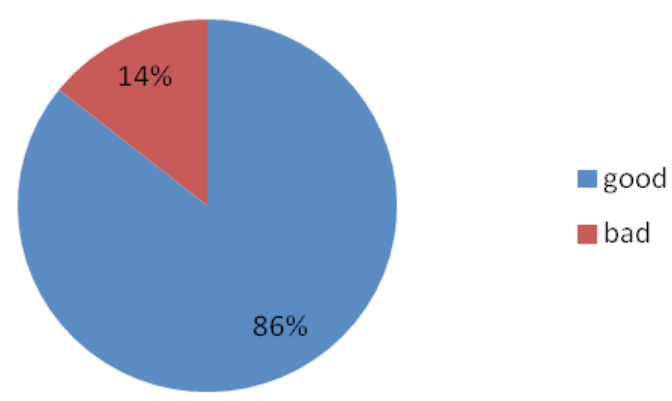

Figure 10. Compliance of patients after treatment.

\section{Discussion}

Hirsutism, acne and alopecia are clinical expressions of hyperandrogenism, one of the most frequent endocrine disorders in women at reproductive age [21]. A thorough interest in finding the treatment for hyperandrogenism has existed since 1975, when Nardi et al have performed a study on 25 female patients suffering from symptoms of virilization (hirsutism, acne, seborrhea and alopecia). They used as treatment an estrogen-progestogen combinantion: cyproterone acetate and ethinylestradiol. This therapy with antiandrogenic and antiovulatory action did not generate side effects. The results of the study showed that in most cases the symptoms of virilization were significantly reduced [22].

A study done by Karrer-Voegeli et al, included females with signs of hyperandrogenism. The subjects performed a laboratory workup to evaluate hormone measurements and received antiandrogen therapy. The combination of Ethinylestradiol and high-dose cyproterone acetate treatment (with peripheral antiandrogenic effect) had good hormone-suppressive effect. Salivary testosterone showed the most significant decrease after the treatment. This therapy lowered the hirsutism score to $53.5 \%$ of baseline at 1 year, and was also effective in treating acne and alopecia [23].

The two studies mentioned above have similar results with ours, showing significant improvement of the acne lesions and important reduction of the hair loss. The fact that these studies were performed with the same type of medication and on a larger number of patients, recording good results with the tested therapy, reinforce our own study performance, encouraging us to say that the tested medication has a beneficial action on Acne and Androgenetic Alopecia.

Experimental research on the same topic have shown positive results of the therapy and the reduction of virilization signs without quantifying the improvement of lesions. Our study clearly indicates the improvement of acne lesions and scars as well as the rate of stopping hair loss and inducing hair regrowth.

Hairloss treatment with anti-androgen therapy

The goal of the treatment in FPHL is to arrest hair loss progression and stimulate hair regrowth. In order to achieve this goal anti-androgen therapies can be used: the classic anti-androgen receptor antagonists, that prevent testosteron and DHT from binding to their receptors, and the peripheral anti-androgens, which alter androgen levels in the hair follicle. Spironolactone and cyproterone acetate are the best well known androgen receptor antagonists. These two medications are the most commonly used androgens in FPHL patients. Spironolactone is a potassium-sparring diuretic, that acts as an anti-androgenic by reducing the levels of total testosterone and competitively blocking the androgen receptor in target tissues [18].

Pregnancy must be avoided while taking spironolactone because of the risk of feminization of the male fetus [19]. Recent studies performed by Sinclair show that $200 \mathrm{mg}$ /day spironolactone has an effect equal to 100 $\mathrm{mg} /$ day cyproterone acetate, determining in $44 \%$ of the cases reduced hair loss and hair regrowth. In $12 \%$ of the cases it stops the disease progression [24].

Treatment with cyproterone can improve hair growth in patients with FPHL, alone or in combination with ethinylestradiol or spironolactone [25]. Action mechanism of cyproterone acetate: it can directly block AR and decrease testosterone levels by suppressing luteinizing hormone and follicle-stimulating hormone release [26]. Cyproterone has shown efficacy in treating patients with FPHL with both increased and normal androgen levels [27]. It is approved for use in Europe and Canada to treat hirsutism, acne and female alopecia.

Literature states that the "gold standard" treatment of Androgenetic Alopecia in women consists of Minoxidil $2 \%$, which is also the only therapy approved by FDA. One of the few controlled studies of therapies for alopecia in women, was performed by Vexiau P et al, who compared topical Minoxidil 2\% and Cyproterone acetate in the treatment of female alopecia; 33 patients received topical Minoxidil 2\% combined with oral contraceptive and the other 33 received Cyproterone acetate $52 \mathrm{mg}$ 1-day plus Ethinyl oestradiol $35 \mathrm{mg}$ for 20 of every 28 days. The study results showed that Minoxidil treatment was more effective in the absence of clinical and biochemical (laboratory) signs of hyperandrogenism. On the other hand, Cyproterone acetate treatment was more effective when the clinical and biochemical factors favoured a diagnosis of hyperandrogenism [28].

The data provided by this study confirms our own results and demonstrate that the oral contraceptive pill (OCP) therapy has a beneficial effect especially in patients with AGA and other signs of hyperandrogenism. Both in Vexiau's study and in our therapeutic protocol we noticed the decrease of hair loss and increase of hair growth as well as the decrease of other hyperandrogenism signs, such as acne vulgaris.

The results achieved at in our study encourage 
us to believe that the therapy with Cyproterone acetate and Ethinyl oestradiol represents a viable treatment with good results with a clinical efficiency similar to the "gold standard" therapy for AGA.

\section{Acne treatment with anti-androgen therapy}

Some patients do not respond well to conventional therapy and common causes of inadequate responses could be the lack of patient compliance and a far too short course of treatment. Effective treatment is necessary to prevent the devastating effects of acne and its complications.

For mild to moderate acne, topical therapies are applied without hyperandrogenic state. They include benzoylperoxide, retinoids, antibiotics, salicylic acid and azelaic acid. Alternative treatments for moderate to severe acne are long-term antibiotics, keratolytics and retinoids (teratogenicity, pregnancy prevision plan, regular liver function) [20].

Anti-androgen therapy influences acne by modifying one of its main causes, the increased sebum production. A minimum reduction of about $35 \%$ in sebum production is needed in order to get a good therapeutic result. Estrogen can be used to decrease sebum production. It reduces ovarian production of androgens by suppressing gonadotropin release. Hormonal therapy may also reduce comedogenesis. Oral contraceptives also increase hepatic synthesis of sex hormone-binding globulin, determining a decrease in circulating free testosterone [21].

Before prescribing hormonal therapy, an examination of the female patient must be performed and also a urine pregnancy test followed by serum beta-hCG test.

If the patient is already taking a contraceptive pill, the change of the contraceptive pill to the new antiandrogen therapy enables fast treatment of acne as well as an effective contraceptive cover.

Combination of birth control pills have shown efficacy in the treatment of acne vulgaris [29].

Anti-androgen therapy has been a key point for dermatologists for more than 35 years, hoping that a safe oral or topical preparation will become available.

Cyproterone acetate has an anti-androgenic effect by blocking androgen receptors. It also reduces androgen synthesis by a negative feedback effect on the hypothalamopituitary-ovarian axis [21]. Cyproterone acetate is the antiandrogen that has been most extensively investigated and used for treating acne patients. Effective oral forms of cyproteron acetate are available by prescription and this therapy alone provides good results in the treatment of acne [22].

An estrogen is often included in the therapy in order to produce an additional benefic effect in treatment and also to provide full contraceptive protection. Estrogen prevents the menstrual irregularities that would occur if cyproteron acetate were to be administered alone. If administered after conception it can cause feminization of the male fetus and it is best used in combination with an oral contraceptive pill (OCP). It is absolutely contraindicated in patients with liver disease.

Two basic methods of anti-androgen therapy are used in clinical practice:

1. Dianette regimen- a combined preparation of 2 $\mathrm{mg}$ cyproterone acetate with $0.035 \mathrm{mg}$ ethinylestradiol, which is taken for 21 days, starting on the 5th day of the menstrual cycle. A 7-day tablet-free interval follows the end of each course, during which a withdrawal bleeding normally occurs.

2. Hammerstein regimen- consists of $50 \mathrm{mg}$ or 100 $\mathrm{mg}$ cyproterone acetate from days 5 to 14 of the menstrual cycle, and $0.05 \mathrm{mg}$ ethinylestradiol from days 5 to 25 , followed by a 7-day tablet-free interval during which a withdrawal bleeding normally occurs. It is usually reserved to patients with severe signs of virilization [24].

If the female patient does not respond well after three months of conventional therapy, then oral hormonal therapy can be substituted for the antibiotic [30].

Individual studies have shown that both oral contraceptive pills (OCPs) and antibiotics are effective in managing acne vulgaris, but few comparisons of efficacy between the 2 therapies have been performed or published. A meta-analysis conducted by Koo EB et al. included a review of 226 publications over 32 randomized controlled trials. The results have showed that antibiotics are superior to OCPs at 3 month assessment. At 6 months OCPs were equivalent to antibiotics in reducing acne lesions, representing a first-line alternative to systemic antibiotics for long-term acne management in women [31].

As the research mentioned above suggests that the efficacy of the treatments with antibiotics and OCP is similar after 6 months, it is important for the therapy administered for such a long time to have minimum side effects, to make administration possible for the time span prescribed by the doctor. The results of our study show increased patients' compliance and reduced side effects after 3 months of treatment with Cyproterone acetate and Ethynil estradiol. This reinforces the possibility of introducing a long term therapy with significantly improved results on acne lesions.

\section{Side-effects of therapy}

The most important side-effects of ciproterone acetate are: thromboembolic events, breast tension, headaches, nausea; these decrease with duration of therapy [30]. Other reactions: irregular menses, weight growth, diminished libido and depression [18]. Ciproterone acetate can also produce abdominal pains, nausea and vomiting, as well as other gastro-intestinal disorders.

Dysmenorrhea may be reduced by co-administration of an oral contraceptive. Proper evaluation of blood pressure and potassium levels from time to time is appropriate.

In 2002 the Pharmacovigilence Working Party 
disscussed the increased venous thromboembolic (VTE) and arterial thromboembolic (ATE) risks and concluded that the use of this drug should be restricted, VTE could be fatal in $1-2 \%$ of the cases. Risk factors for VTE include history of VTE, pregnancy, trauma, surgery, immobilization (after surgery or long flights), obesity and smoking (prothrombotic state), and hereditary thrombophilic defects. The risk of VTE is highest during the first year a woman starts hormonal contraceptives or when she re-starts after a period of non-use of at least one month [32]. After the first year, the risk drops to a constant lower level. CPA/EE (2 $\mathrm{mg} / 0.035 \mathrm{mg}$ ) should be contraindicated in patients with a history or hereditary predisposition of venous thrombosis. They should not be given at the same time with other hormonal contraceptives. The duration of the treatment is at least three months with a periodical evaluation to continue the treatment [23].

\section{Conclusion}

This study allowed us to establish the efficacy of Melleva 35 therapy on acne and alopecia, both from the doctor's and patient's point of view; $83 \%$ of study subjects reported their hair did not continue to fall after the 3 months antiandrogen therapy regimen. More than half of the study subjects (54\%) declared that hair loss partially stopped right after the first month of therapy. The females were evaluated using trichoscopy and the doctor noticed hair regrowth in $77 \%$ of the cases.

Regarding the improvement of acne lesions after the treatment, $40 \%$ of study subjects reported good improvement and $26 \%$ reported excellent results with Melleva 35. There was a significant improvement in the majority of female patients, the number of the acne lesions (pustules and papules) and inflammatory acne were reduced. Melleva therapy worked better on the inflammatory acne lesions than on the scars, as in $60 \%$ of study subjects there was no improvement of the scar aspect after the treatment.

The acceptance of the treatment was very high, $86 \%$ patients were compliant with the study therapy: Melleva 35 , one pill per day, for three consecutive months. Only 5 females had bad compliance to the treatment, due to adverse reactions: abdominal pain (3 subject), nausea ( 1 subject) and vomiting (1 subject). The rate of adverse events was within the limits of the treatment tested by the study. Tolerance was good and very good in $80 \%$ of the cases, with almost an equal distribution between the specified categories.

Almost a third of the total number of subjects (28.5\%) reached a good satisfaction level after the treatment, while $37.1 \%$ claimed moderate satisfaction.

There was no correlation between the age of the subjects and the treatment for acne therefore our first hypothesis was rejected.

As a conclusion, antiandrogenic therapy with
Melleva 35, one pill per day, for 3 consecutive months, shows good results for patients who suffer from both Androgenetic Alopecia and Acne Vulgaris.

\section{References:}

1. Mc Phee SJ, Papadachis MA, Tierney LM. Current medical diagnosis and treatment. 46th Edition. Mc Hill Companies: Lange; 2007.

2. Orasan MS, Bondor CI, Coneac A, Mitrea DR, Orasan RI, Muresan A. Factors that influence the psychosocial status of patients with female pattern hair loss. Paripex Indian J Res. 2013; 2(8):48-51.

3. Burgdorf WH, Plewig G, Wolff HH, Landthaler M. BraunFalco's Dermatology. 3rd edition. Springer; 2009.

4. Fenstein Robert P. Androgenic Alopecia. Medscape Drugs \& Diseases, updated Sep. 8, 2014. Avalaible from: http://emedicine. medscape.com/article/1070167-overview\#a0199.

5. Adityan B, Kumari R, Thappa DM. Scoring systems in acne vulgaris. Indian J Dermatol Venereol Leprol. 2009;75:323-326.

6. Thiboutot D, Gollnick H, Bettoli V, Dréno B, Kang S, Leyden JJ, et al. New insights into the management of acne: an update from the Global Alliance to Improve Outcomes in Acne group. J Am Acad Dermatol. May 2009;60(5 Suppl):S1-50.

7. Forsea D, Popescu R, Popescu CM. Compendiu de Dermatologie si Venerologie [Compendium of Dermatology and Venerology]. Bucuresti: Ed Tehnica; 1996.

8. Patel F, Cuniffe WJ. Key facts about acne. London: Mediscript; 1993.

9. Kariya Y, Moriya T, Suzuki T, Chiba M, Ishida K, Takeyama J, et al. Sex steroid hormone receptors in human skin appendage and its neoplasms. Endocr J. 2005;52(3):317-325.

10. Pochi PE, Strauss JS. Sebaceous gland response in man to the administration of testosterone, delta-4-androstendione and dehydroisoandrosterone. J iNvest Dermatol. 1969;52(1):32-36.

11. Harris HH, Downing DT, Stewart ME, Strauss JS. Sustainable rates of sebum secretion in acne patients and matched normal control subjects. J Am Acad Dermatol. 1983;8:200-203.

12. Thiboutot D, Gilliland K, Light J, Lookingbill D. Androgen metabolism in sebaceous glands from subjects with and without acne. Arch Dermatol. 1999;135(9):1041-1045.

13. Levell MJ, Carwood ML, Burke B et al. Acne is not associated with abnormal plasma androgens. Br J Dermatol. 1989;120(5):649-654.

14. Lookingbill DP, Horton R, Demers LM, Egan N, Marks JG Jr, Santen RJ. Tissue production of androgens in women with acne. J Am Acad Dermatol. 1985;12(3):481-487.

15. Chen W, Thiboutot D, Zouboulis C. Cutaneous androgen metabolism: basic research and cinical perspectives. J Invest Dermatol. 2002;119:992-1007.

16. Orasan MS. Physiopathological factors and therapeutic options in females with androgenetic alopecia. $\mathrm{PhD}$ thesis. Ed. Med. Iuliu Hatiaganu Cluj. 2013;(1):24-25.

17. Orasan MS, Coneac A, Orasan RI. Non-invasive methods of evaluation in female pattern hair loss: Common problems in Clinical practice. Indian J Appl Res. 2013;3(9):12-16.

18. Shapiro J. Preface to: Hair disorders: Current Concepts in Physiopathology, Diagnosis and Management, 2013 Elsevier, Dermatology Clinics. 2013:31XIII.

19. Coursen P, Messenger A. Female pattern hair loss in complete androgen insensitivity syndrome. $\mathrm{Br}$ J Dermatol. 2010;162 


\section{Dermatology}

(5):1135-1137.

20. Futterweit W, Dunaif A, Yeh HC, Kingsley P. The prevalence of hyperandrogenism in 109 consecutive female patients with diffuse alopecia. J Am Acad Dermatol. 1988;19:831-836.

21. Gollnick H, Cunliffe W, Berson D, Dreno B, Finlay A, Leyden JJ, et al. Management of acne: a report from a Global Alliance to Improve Outcomes in Acne. J Am Acad Dermatol. 2003;49 (1 Suppl):S1-37.

22. Nardi M, De Aloysio D, Busacchi P, Flamigni C. Cyproterone acetate - ethinylestradiol treatment of hirsutism, acne, seborrhea and alopecia. Acta Eur Fertil. 1975;6(2):153-165.

23. Karrer-Voegeli S, Rey F, Reymond MJ, Meuwly JY, Gaillard RC, Gomez F. Androgen dependence of hirsutism, acne, and alopecia in women: retrospective analysis of 228 patients investigated for hyperandrogenism. Medicine (Baltimore). 2009;88(1):32-45.

24. Sinclair R. Leerinke M, Jolley D. Treatment of female pattern hair loss with oral antiandrogens. Br J Dermatol. 2005; 152:466-473. 25. Rogers NE, Abram MR. Medical treatments for male and female pattern hair loss. J Am Acad Dermatol. 2008;59(4): 547 566.

26. Camacio-Martines FM. Hair loss in women. Semin Cutan
Med Surg. 2009;28:19-32.

27. Karrer-Voegeli S, Rey F, Reymond MJ, Meuwly JY, Gaillard RC, Gomez F. Androgen dependence of hirsutism, acne and alopecia in women: a prospective analysis of 228 patients investigated for hyperandrogenism. Medicine(Baltimore). 2009;88(1):32-45.

28. Vexiau P, Chaspoux C, Boudou P, Fiet J, Jouanique C, Hardy N, et al. Effects of minoxidil 2\% vs. cyproterone acetate treatment on female androgenetic alopecia: a controlled, 12-month randomized trial. Br J Dermatol. 2002;146(6):992-999.

29. Koulianos GT. Treatment of acne with oral contraceptives: criteria for pill selection. Cutis. 2000;66(4):281-286.

30. Cunliffe WJ. A brief guide to the Management and Treatment of acne. Shering, 1991

31. Koo EB, Petersen TD, Kimball AB. Meta-analysis comparing efficacy of antibiotics versus oral contraceptives in acne vulgaris. J Am Acad Dermatol. 2014;71(3):450-459.

32. Dinger JC, Heinemann LA, Kühl-Habich D. The safety of a drospirenone-containing oral contraceptive: final results from the European Active Surveillance Study on oral contraceptives based on 142,475 women-years of observation. Contraception. 2007;75(5):344-354. 